\title{
Second-Look Arthroscopic Assessment and Clinical Results of Modified Pull-Out Suture for Posterior Root Tear of the Medial Meniscus
}

\author{
Jin-Ho Cho, MD and Jae-Gwang Song, MD \\ Department of Orthopaedic Surgery, Inje University Ilsan Paik Hospital, Goyang, Korea
}

\begin{abstract}
Purpose: To identify the structural integrity of the healing site after arthroscopic repair of a posterior root tear of the medial meniscus by second-look arthroscopy and to determine the clinical relevance of the findings.

Materials and Methods: From January 2005 to December 2010, 20 consecutive patients underwent arthroscopic modified pull-out suture repair for a posterior root tear of the medial meniscus. Thirteen patients were available for second-look arthroscopic evaluation. The healing status of the medial meniscus was classified as complete healing, lax healing, scar tissue healing, and failed healing. We evaluated the correlation between the clinical symptoms and second-look arthroscopic findings. Clinical evaluation was based on the Lysholm knee scores and Hospital for Special Surgery (HSS) scores.

Results: There were 4 cases of complete healing, 4 lax healing, 4 scar tissue healing, and 1 failed healing. The healing status of the repaired meniscus appeared to be related to the clinical symptoms. Patients who achieved complete tissue healing had no complaint. The healing status exhibited no relationship with age, mechanical axis, degree of subluxation, and symptom duration. The mean Lysholm score improved from 34.7 preoperatively to 75.6 at follow-up and the mean HSS score also significantly increased from 33.5 to 82.2.

Conclusions: We achieved 4 complete and 8 partial healing (lax or scar) of the medial meniscus in this retrospective case series of posterior horn meniscus root repairs performed by 1 surgeon. Further research is needed to clarify why all patients showed clinical improvement despite findings of partial healing on second-look arthroscopy.
\end{abstract}

Keywords: Knee joint, Root tear, Medial meniscus, Posterior horn, Arthroscopy, Modified pull out suture

\section{Introduction}

Transection of the posterior horn of the medial meniscus destroys the ability of the meniscus to withstand hoop tension. As a result, extrusion of the medial meniscus from the joint space occurs; this can then lead to the development of a medial compartment degenerative arthritis ${ }^{1,2)}$. For complete radial tears of the

Received July 23, 2013; Revised November 6, 2013;

Accepted January 15, 2014

Correspondence to: Jin-Ho Cho, MD

Department of Orthopaedic Surgery, Inje University Ilsan Paik Hospital, 170 Juhwa-ro, Ilsanseo-gu, Goyang 411-706, Korea

Tel: +82-31-910-9733, Fax: +82-31-910-7967

E-mail: osd07@paik.ac.kr

This is an Open Access article distributed under the terms of the Creative Commons Attribution Non-Commercial License (http://creativecommons.org/licenses/by-nc/3.0/) which permits unrestricted non-commercial use, distribution, and reproduction in any medium, provided the original work is properly cited. meniscal posterior horn, pull out suture repair or suture anchor repair of the meniscus may be used to restore the hoop tension of the meniscus. Biomechanical studies recently demonstrated that the repair of a posterior root tear of the medial meniscus could restore knee joint kinematics in cadaver knees ${ }^{3,4)}$. However, few clinical investigations have been performed to determine the outcomes of root repair of the medial meniscus ${ }^{5}$.

The objective of this study was to determine the structural integrity of the meniscal healing site with second-look arthroscopy and to evaluate the clinical relevance of these findings after arthroscopic repair of root tears of the medial meniscus.

\section{Materials and Methods}

\section{Patient Selection}

After obtaining approval from our institutional review board, we conducted a retrospective review of medical records of patients who underwent arthroscopic pull-out suture repair for a 
root tear of the medial meniscus at our institution between January 2005 and December 2010. The indications for surgery were as follows: 1) an acute traumatic or chronic degenerative root tear based on clinical and magnetic resonance imaging (MRI) findings, 2) Kellgren-Lawrence grade 1 or 2 on preoperative simple radiographs, 3) International Cartilage Repair Society (ICRS) grade II or less chondral lesion according to preoperative MRI, 4) less than $4.5 \mathrm{~mm}$ medial meniscus subluxation from the medial tibial plateau on coronal MRI, and 5) weight bearing line (the line connecting the center of the femoral head to the center of the ankle) passing more than $25 \%$ of the tibial width in lower extremity scanography. Potentially irreparable complex root tears and root tears with definitive medial meniscus degeneration were excluded because the meniscus was not considered sufficiently strong to withstand the tension after sutures due to severe degeneration. In addition, patients with a body mass index (BMI) of greater than 30 were excluded. Patients with other procedures were included (articular cartilage debridement in 2 and Baker's cystectomy in 1). We evaluated the age, weight bearing line (mechanical alignment), symptom duration, meniscus subluxation on MRI to investigate the factors related to healing potential. Among the 20 consecutive cases, 13 underwent second look arthroscopy and were thus enrolled in this retrospective study. All data were analyzed, including information obtained from reviewing medical records and arthroscopic photographs. Second-look arthroscopy was performed after hardware removal on patient's demand 6 months after surgery.

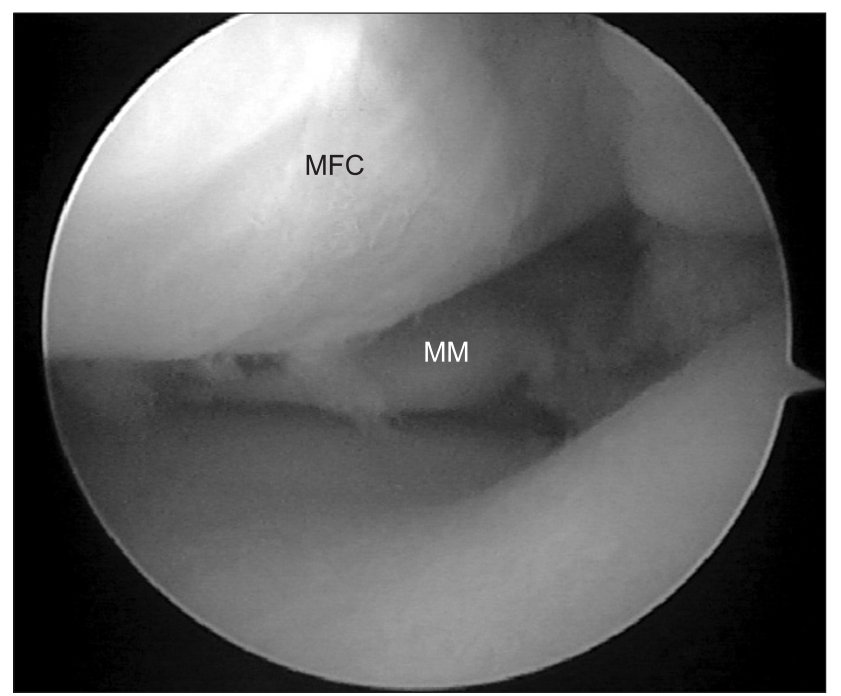

Fig. 1. Arthroscopic image showing a complete root tear of the posterior horn of the medial meniscus (MM) through the anterolateral portal in the left knee. MFC: medial femoral condyle.

\section{Surgical Technique}

Modified pull-out suture repair was performed using a technique described by $\mathrm{Cho}^{6}{ }^{6}$. After routine arthroscopic examination of the knee joint, a posteromedial portal was established using the transillumination technique. After identifying the location of a lesion (Fig. 1), the footprint was confirmed by removing the cartilage of the tibial plateau that was attached to the posterior root of the medial meniscus, using a curette and a round bur inserted through the posteromedial portal. Under arthroscopic visualization through the anterolateral portal, the anterior cruciate ligament tibial drilling guide (Linvatec, Largo, FL, USA) was introduced through the posteromedial portal. The tip of the guide was placed at the decorticated footprint of the posterior root of the medial meniscus. After making a $2-\mathrm{cm}$ vertical incision on the anteromedial cortex of the proximal tibia, the sleeve of the guide with $40^{\circ}-45^{\circ}$ was fixed. The entry point at the tibial anteromedial cortex was aimed at the midportion of the tibial shaft and approximately $2-3 \mathrm{~cm}$ anterior to the medial collateral ligament insertion. A guide pin was drilled through the sleeve from the anteromedial cortex of the proximal tibia to the posterior root tear site of the medial meniscus. A tibial tunnel was made using a $6 \mathrm{~mm}$ reamer (Linvatec) to extend from the anteromedial cortex of the proximal tibia to the footprint of the posterior root of the medial meniscus. While visualizing from the anterolateral portal, a crescent-shaped suture hook (Linvatec) loaded with a No. 0 PDS (Ethicon, Somerville, NJ, USA) suture material was inserted through the posteromedial portal (Fig.

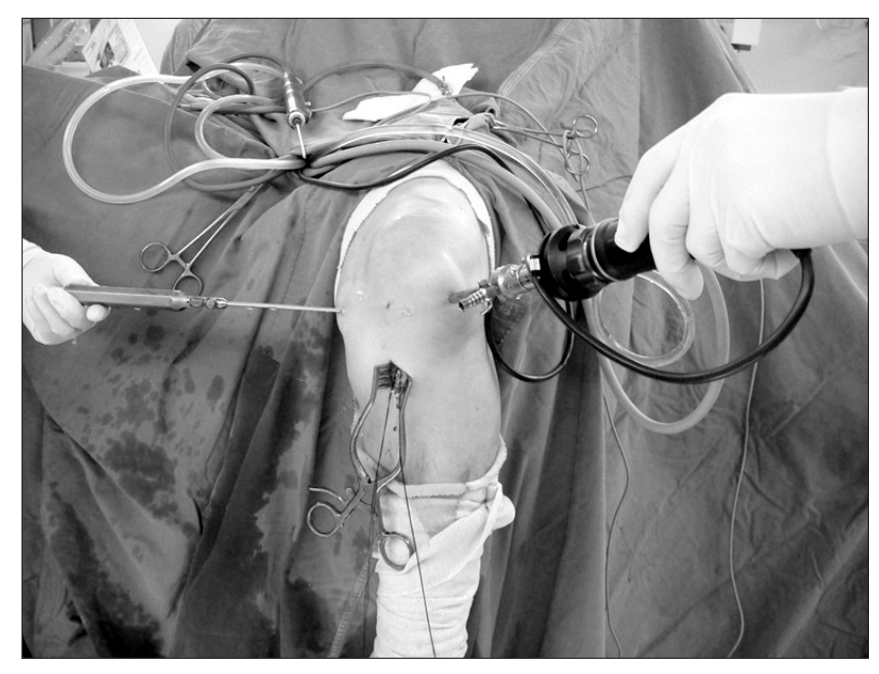

Fig. 2. Under visualization through the anterolateral portal, a suture hook loaded with a No. 0 PDS suture material was inserted through the posteromedial portal. With a grasper, it was advanced into the intraarticular space through the tibial tunnel, and one end of the strand was retrieved out of the tunnel. 


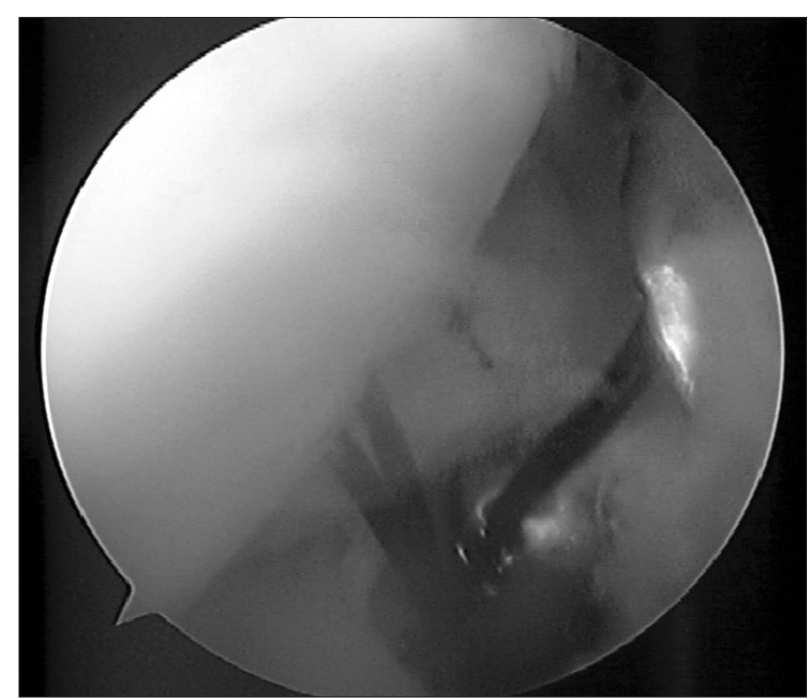

Fig. 3. Arthroscopic image showing the suture hook tip penetrating through the detached root portion of the medial meniscus posterior horn from the femoral surface to the tibial surface in a vertical direction. After one end of the PDS strand was advanced through the suture hook, it was retrieved out through the transtibial tunnel using an already inserted grasper.

2). The detached root portion of the medial meniscus posterior horn was penetrated by the sharp tip of the crescent-shaped suture hook from the femoral surface to the tibial surface of the meniscus in a vertical direction. Then, some portion of the total length of the PDS was advanced through the suture hook into the intra-articular space through the tibial tunnel with a grasper. At the same time, the one end of the suture was retrieved through the tibia using the already inserted grasper (Fig. 3), and the suture hook was withdrawn upward. Subsequently, the other end of the PDS strand was retrieved through the tibial tunnel with a grasper. In the same manner, additional 1 PDS suture strand was advanced through the posterior horn of the medial meniscus into the tibial tunnel with a grasper. By pulling the ends of the suture under adequate tension, the posterior root of the medial meniscus could be reduced and stabilized under adequate tension (Fig. 4). Subsequently, the suture strands were post-tied and fixed with a $6.5 \mathrm{~mm}$ a cancellous bone screw and a smooth washer to the anterior cortex of the tibia.

\section{Postoperative Management}

A cylinder leg splint was applied with the leg in full extension for 2 postoperative weeks and a limited-motion brace was subsequently applied to restrict knee motion. Patients were kept nonweight bearing on crutches for 6 postoperative weeks. Quadriceps muscle exercises, as well as straight-leg raise exercises, were

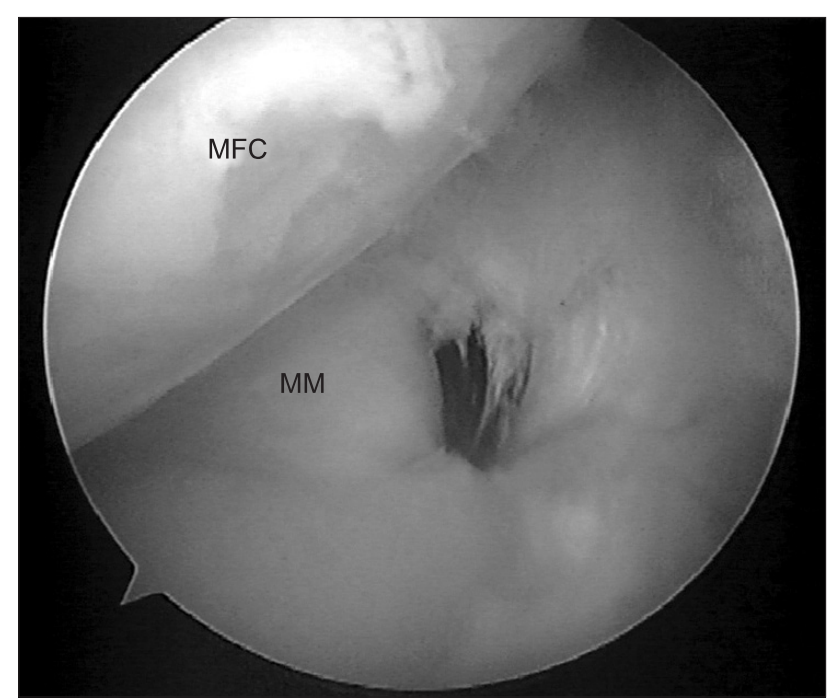

Fig. 4. Arthroscopic image from the anterolateral portal showing reattachment of the posterior root of the medial meniscus to the tibial tunnel site under adequate tension. MM: medial meniscus, MFC: medial femoral condyle.

performed several times daily. Up to $90^{\circ}$ of active motion was allowed after the first 4 weeks and then the range of flexion increased gradually up to $135^{\circ}$. Full-weight bearing without crutches was allowed 8 weeks after surgery. Full flexion and squatting were permitted 3 months after surgery. Patients returned to their previous level of exercise after 6 postoperative months.

\section{Second-Look Arthroscopy}

Among the 20 patients who underwent root repair, 13 were available for second-look arthroscopy performed by one orthopaedic surgeon at our hospital. The healing status of the repaired meniscus was classified according to the method of Seo et al. ${ }^{5)}$ (complete healing, lax healing, scar tissue healing, and failed healing). Complete healing was defined as meniscal continuity with no cleft, no lifting on probing, and normal meniscal tension at the repair site (Fig. 5). Lax healing was defined as apparent increase in meniscus lifting and mobility on probing with good meniscal continuity (Fig. 6). Scar tissue healing was defined as a meniscus that could be easily raised on probing and showed no true meniscal continuity except for some connecting scar tissue fibers between the tibial attachment site and the posterior horn of the medial meniscus (Fig. 7). Finally, failed healing was defined as no continuity and no evidence of meniscal healing at the repair site (Fig. 8). In addition, chondral lesions were evaluated during second-look arthroscopy using arthroscopic photographs and described according to ICRS grade ${ }^{7}$. 

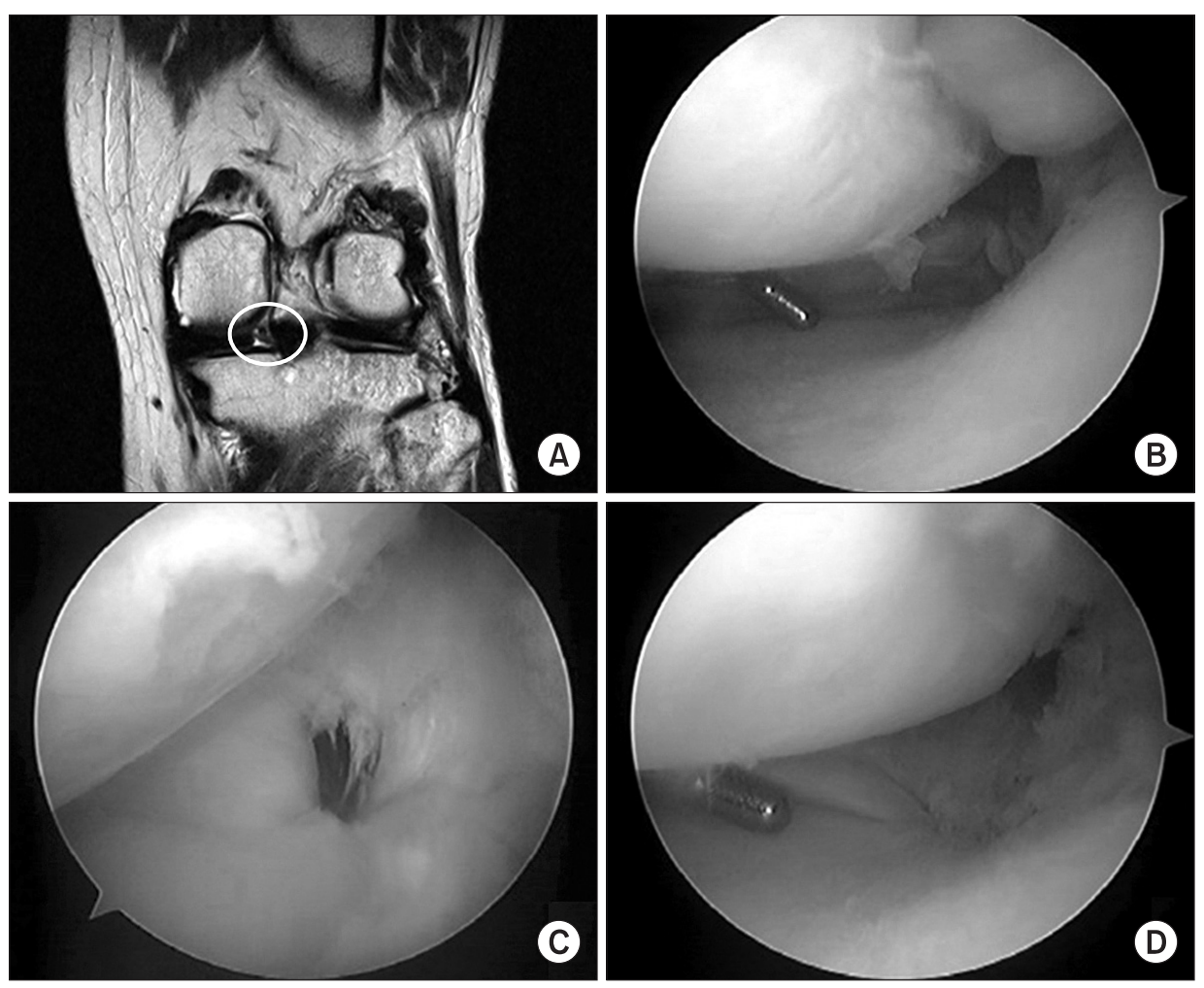

Fig. 5. Images of the knee with complete healing. (A) T2-coronal magnetic resonance image showing a root tear of the posterior horn of the medial meniscus in the left knee. (B) Arthroscopic photograph showing the complete root tear of the posterior horn of the medial meniscus. (C) Arthroscopic photograph showing firm reattachment of the posterior root of the medial meniscus to the tibial tunnel site. (D) Second look arthroscopic photograph showing complete healing of the root tear site of the medial meniscus.
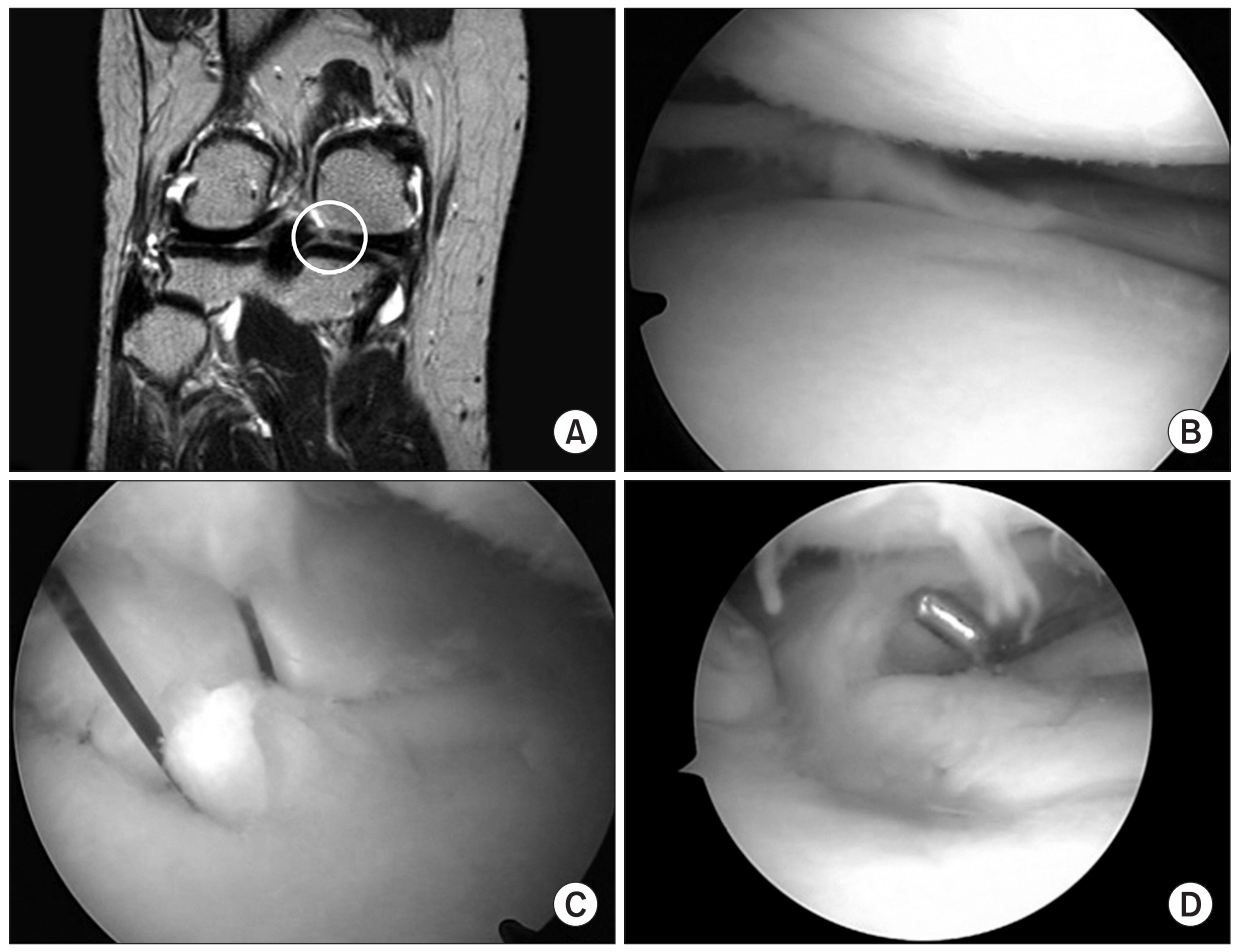

Fig. 6. Images of the knee with lax healing. (A) T2-coronal magnetic resonance image showing a root tear of the posterior horn of the medial meniscus in the right knee. (B) Arthroscopic photograph showing the complete root tear of the posterior horn of the medial meniscus. (C) Arthroscopic photograph showing reattachment of the posterior root of the medial meniscus to the tibial tunnel site. (D) Second look arthroscopic photograph showing lax healing of root tear site of the medial meniscus; it was easily lifted on probing despite good meniscal continuity.

\section{Clinical Assessment}

Clinical assessment consisted of evaluation using objective and subjective Lysholm functional questionnaires and Hospital for
Special Surgery (HSS) scores performed before surgery and at the final follow-up. Final follow-up examinations were performed immediately before second-look arthroscopy. Symptomatic im- 

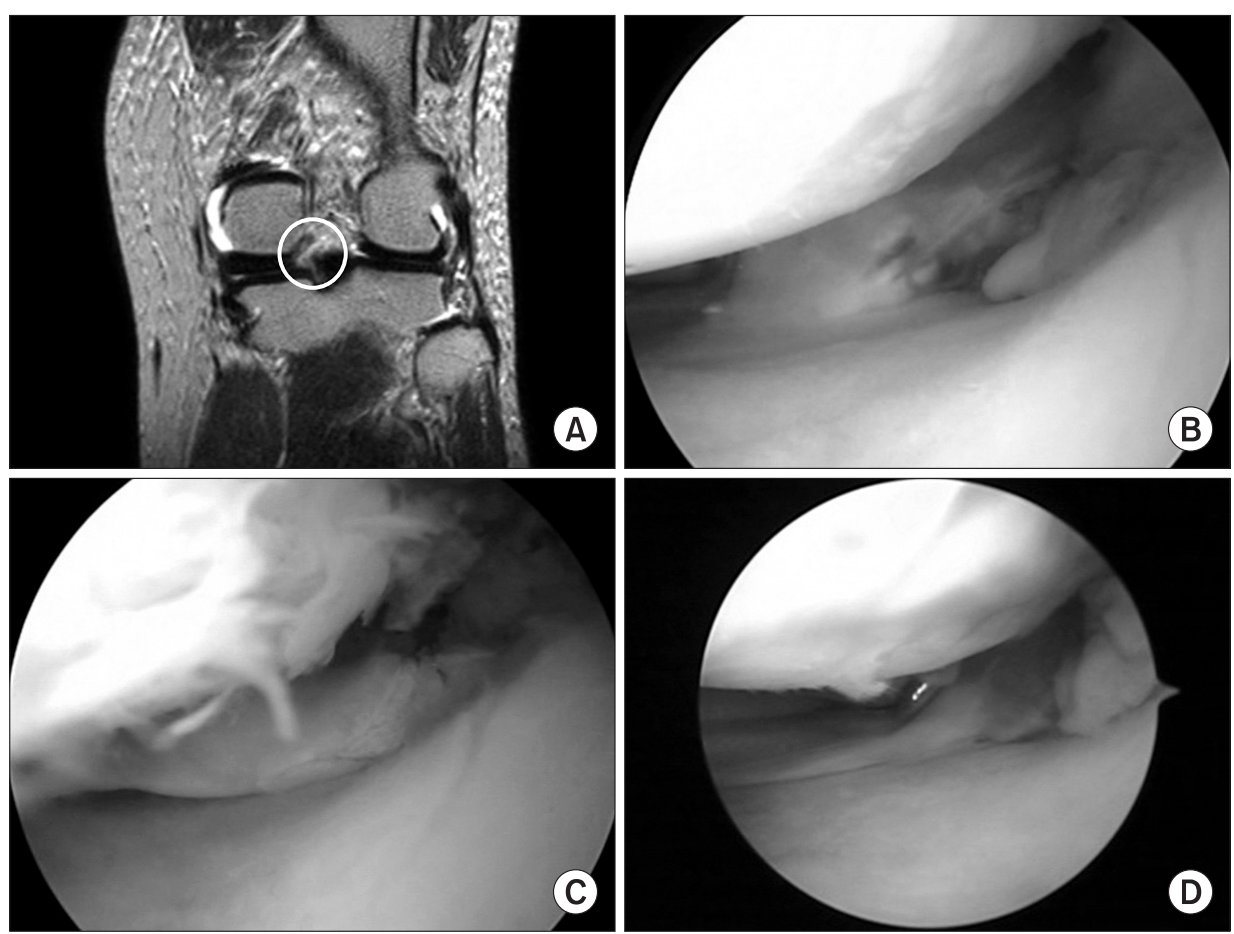

(B)

(D)
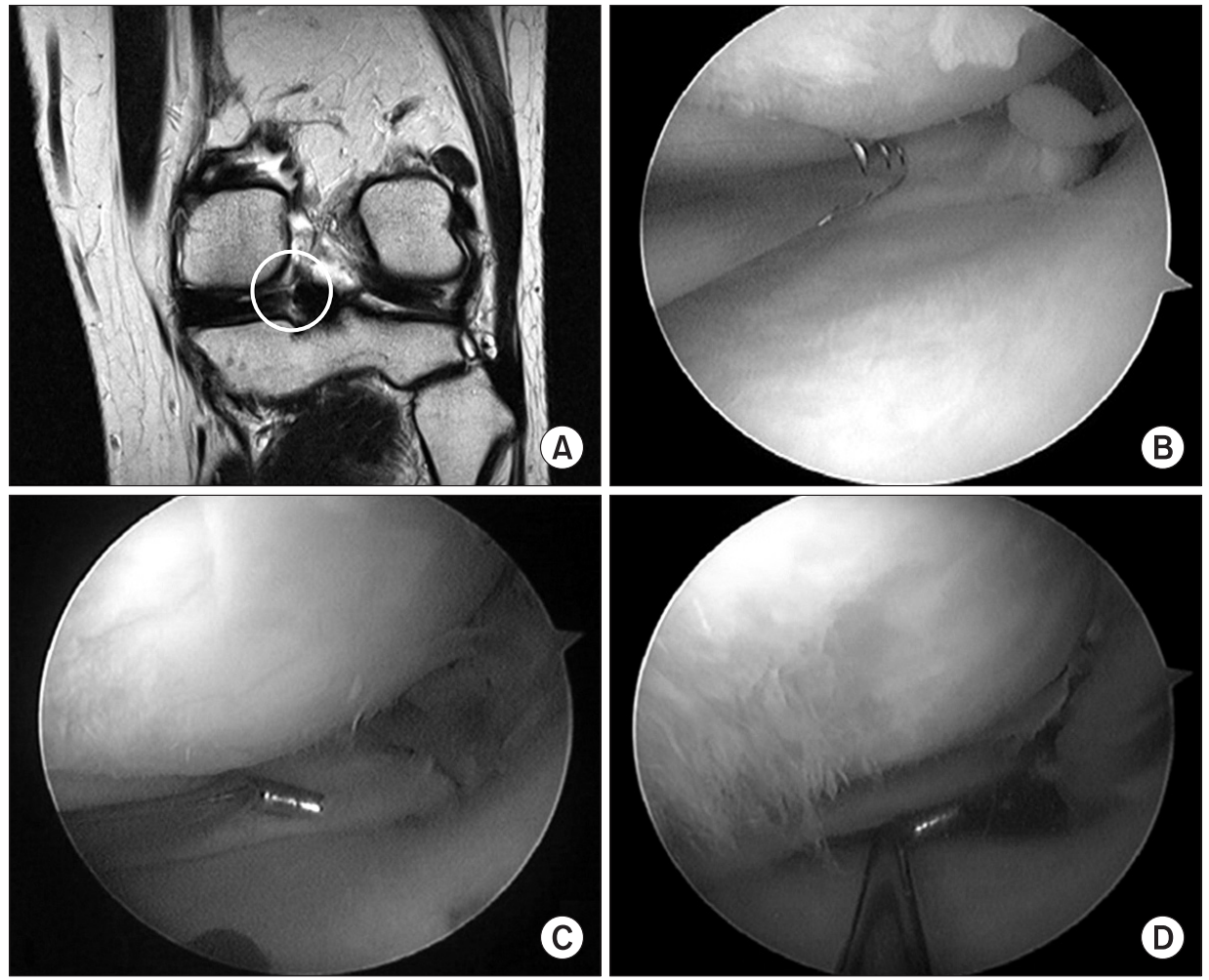

Fig. 8. Images of the knee with failed healing. (A) T2-coronal magnetic resonance image showing a root tear of the posterior horn of the medial meniscus in the left knee. (B) Arthroscopic photograph showing the complete root tear of the posterior horn of the medial meniscus. (C) Arthroscopic photograph showing reattachment of the posterior root of the medial meniscus to the tibial tunnel site. (D) Second look arthroscopic photograph showing no continuity and no evidence of meniscal healing at the repair site; it was easily lifted with a probe. provement after pull-out suture was evaluated at the final followup. Statistical analysis was performed with SPSS 11.0. (SPSS Inc., Chicago, IL, USA) The Wilcoxon signed-rank test was used to compare preoperative and postoperative data. Statistical significance was accepted at $\mathrm{p}<0.05$. 


\section{Results}

Among the 13 patients who were available for second-look arthroscopy, 12 were female and 1 was male. Their average age at the time of the index surgery was 50.3 years (range, 41 to 69 years). The mean symptom duration before root repair was 12.5 weeks (range, 1 week to 18 months), and the average time between the initial surgery and the second-look arthroscopy was 7.1 months (Table 1). All cases had no acute trauma events.

\section{Second-Look Arthroscopy}

Among the 13 patients, 7 were asymptomatic. The remaining 6 complained of knee pain aggravated by going down stairs and showed joint line tenderness and effusions at physical examination without any specific signs associated with mechanical ab- normalities, such as locking, giving way and limitation of range of motion. At second-look arthroscopy, complete tissue healing with symptomatic improvement was seen in four knees, lax tissue healing in four knees (symptomatic in 2 and asymptomatic in 2), scar tissue healing in four knees (symptomatic in 3 and asymptomatic in 1) and failed healing with no symptomatic improvement in 1 knee. Two patients who underwent chondral shaving of the medial femoral condyle (due to ICRS grade 2) achieved complete tissue healing and no aggravation of the cartilage lesion in the medial femoral condyle. The healing status of the repaired meniscus appeared to be related to the postoperative clinical symptoms. The four patients who achieved complete tissue healing were all asymptomatic. Two of four patients who achieved lax tissue healing and three of the four patients who achieved scar tissue healing and one who failed tissue healing complained of per-

Table 1. Patient Demographics and General Findings

\begin{tabular}{|c|c|c|c|c|c|c|c|c|c|c|c|}
\hline No. & Sex & $\begin{array}{l}\text { Age } \\
(\mathrm{yr})\end{array}$ & $\begin{array}{l}\text { Injured } \\
\text { knee }\end{array}$ & $\begin{array}{c}\text { WBL } \\
(\%)\end{array}$ & $\begin{array}{l}\text { Meniscal } \\
\text { subluxation } \\
\quad(\mathrm{mm})\end{array}$ & $\begin{array}{l}\text { Symptom } \\
\text { duration } \\
\text { (wk) }\end{array}$ & $\begin{array}{l}\text { Cartilage } \\
\text { findings }\end{array}$ & Additional procedures & $\begin{array}{c}\text { Symptom } \\
\text { improvement }\end{array}$ & $\begin{array}{l}\text { F/U duration } \\
(\mathrm{mo})\end{array}$ & $\begin{array}{l}\text { Healing } \\
\text { status }\end{array}$ \\
\hline 1 & $\mathrm{~F}$ & 56 & $\mathrm{~L}$ & 33 & 3.8 & 4 & II/II & None & No & 24 & Scar \\
\hline 2 & M & 47 & $\mathrm{~L}$ & 35 & 1.7 & 7 & $\mathrm{II} / \mathrm{II}$ & Articular cartilage debridement & No & 6 & Failed \\
\hline 3 & $\mathrm{~F}$ & 56 & $\mathrm{~L}$ & 42 & 1.2 & 8 & $\mathrm{I} / \mathrm{I}$ & None & Improve & 9 & Complete \\
\hline 4 & $\mathrm{~F}$ & 56 & $\mathrm{~L}$ & 25 & 0 & 36 & II/III & None & No & 8 & Lax \\
\hline 5 & $\mathrm{~F}$ & 41 & $\mathrm{~L}$ & 55 & 0 & 4 & $\mathrm{I} / \mathrm{I}$ & None & Improve & 6 & Complete \\
\hline 6 & $\mathrm{~F}$ & 46 & $\mathrm{R}$ & 43 & 2.5 & 2 & II/II & None & Improve & 9 & Complete \\
\hline 7 & $\mathrm{~F}$ & 64 & $\mathrm{R}$ & 26 & 1.8 & 1 & II/II & Articular cartilage debridement & Improve & 7 & Complete \\
\hline 8 & $\mathrm{~F}$ & 47 & $\mathrm{~L}$ & 44 & 2.2 & 8 & II/II & None & No & 5 & Scar \\
\hline 9 & $\mathrm{~F}$ & 55 & $\mathrm{R}$ & 27 & 3.2 & 8 & II/II & Baker's cystectomy & Improve & 7 & Lax \\
\hline 10 & $\mathrm{~F}$ & 57 & $\mathrm{R}$ & 67 & 2.8 & 1 & II/II & None & No & 5 & Scar \\
\hline 11 & $\mathrm{~F}$ & 56 & $\mathrm{R}$ & 28 & 4.2 & 72 & II/II & None & Improve & 6 & Scar \\
\hline 12 & F & 68 & $\mathrm{~L}$ & 39 & 1.4 & 4 & II/II & None & No & 6 & Lax \\
\hline 13 & $\mathrm{~F}$ & 48 & $\mathrm{R}$ & 56 & 4.3 & 8 & II/II & None & Improve & 6 & Lax \\
\hline
\end{tabular}

WBL: weight bearing line, F/U: follow-up, L: left, R: right.

Table 2. Factors Associated with Healing Status

\begin{tabular}{lccccc}
\hline \multicolumn{1}{c}{ Variable } & Complete & Lax & Scar & Fail & p-value \\
\hline $\mathrm{N}$ & 4 & 4 & 4 & 1 & \\
Age (yr) & 51.75 & 56.75 & 54 & 47 & $>0.05$ \\
WBL (\%) & 41.33 & 37.75 & 40 & 35 & $>0.05$ \\
Subluxation (mm) & 1.37 & 3.17 & 2.3 & 1.7 & $>0.05$ \\
Duration (wk) & 3.75 & 24 & 44 & 7 & $>0.05$ \\
Symptom improvement (\%) & 100 & 71 & 25 & 0 & $<0.05$ \\
\hline
\end{tabular}

WBL: weight bearing line.
Table 3. Functional Score Comparison

\begin{tabular}{lccccc}
\hline \multirow{2}{*}{ Variable } & \multicolumn{2}{c}{ Lysholm score } & & \multicolumn{2}{c}{ HSS score } \\
\cline { 2 - 3 } \cline { 5 - 6 } & Preop & Postop & & Preop & Postop \\
\hline Complete & 35.4 & 83.2 & & 31.6 & 87.7 \\
Lax & 32.2 & 77.5 & & 36.8 & 85.3 \\
Scar & 37.6 & 71.5 & & 34.3 & 78.4 \\
Fail & 31 & 54 & & 25 & 63 \\
Total & 34.8 & 75.6 & & 33.5 & 82.2 \\
\hline
\end{tabular}

HSS: Hospital for Special Surgery, Preop: preoperative, Postop: postoperative. 
sistent knee pain (Table 1). However, the healing status showed no relationship with age, BMI, symptom duration, Mechanical alignment and the degree of medial meniscus subluxation (Table 2).

\section{Clinical Assessment}

The mean Lysholm score increased from 34.7 points (range, 31 to 40 points) before root repair to 75.6 points (range, 51 to 89 points) at the second-look arthroscopy $(\mathrm{p}<0.5)$. Similarly, the mean Hospital for Special Surgery score also significantly improved from 33.5 points (range, 25 to 46 points) to 82.2 points (range, 63 to 95 points; $\mathrm{p}=0.003$ ) The Clinical scores of the complete tissue healing group were higher than those of the lax, scar or failed healing groups (Table 3).

\section{Discussion}

Recently, posterior root tears of the medial meniscus have become increasingly recognized, but relatively few reports have described the results of meniscal tear repairs. Traditionally, partial menisectomy has been considered an effective treatment for posterior root tears of the medial meniscus ${ }^{8,9)}$. Bin et al. ${ }^{10)}$ also achieved good results using a partial menisectomy in patients in whom a posterior root tear of the medial meniscus was the main cause of mechanical pain. However, it has been recognized that while some of these patients do well after surgery, many patients continue to suffer from knee pain during weight-bearing activities and are dissatisfied with the outcomes of the procedure. Partial menisectomy does not restore the normal biomechanical function of the meniscus. In addition, root repairs have yet to be proven to ensure normal kinematics in in vivo human studies. It is believed that a torn medial meniscus root should be repaired to recover hoop tension, even though the posterior horn attachment of the meniscus is covered with vascular synovial tissues and appears to have a good blood supply ${ }^{11}$.

Many surgical techniques have recently been introduced for the repair of posterior root tears of the medial meniscus ${ }^{12-14)}$. Repair is a reasonable option in acute and subacute cases or in chronic cases where there is little or no articular cartilage but good preservation of healthy meniscal tissue. Griffith et al. ${ }^{15)}$, described a technique that combines an arthroscopic approach to repair the medial meniscal root to a decorticated foot print via suture tunnels over a bone bridge. Ahn et al. ${ }^{16)}$ recommended a pullout suture technique for anchoring the posterior horn of the meniscus. Lee et al. ${ }^{17)}$ demonstrated that clinical results significantly im- proved at a minimum follow-up of 2 years after the arthroscopic pullout suture repair of posterior root tears of the medial meniscus. They also reported that all repaired menisci had healed completely by second-look arthroscopy without additional chondral lesions. On the other hand, Seo et al. ${ }^{5)}$ reported that none of the 11 knees showed complete meniscus healing at the final followup. The majority showed lax or scar tissue healing, even though meniscal continuity was observed between the posterior horn of the medial meniscus and the tibial attachment site on the secondlook arthroscopy.

We used an all-arthroscopic modified pull-out suture technique in an attempt to restore the meniscal attachment in the present study. Four of the 13 knees showed complete meniscus healing. Compared with the other healing status groups (lax, scar, and failed healing groups), there were no specific factors that could be associated with the meniscal healing in the complete healing group. However, the 1 patient with failed meniscal healing was not cooperative with the postoperative care. He did not sufficiently adhere to non-weight bearing restrictions. We think that a sufficient period of non-weight bearing restriction is crucial to the meniscal healing. From a clinical point of view, significant improvements were obtained based on the evaluation of the Lysholm scores and HSS scores. Seo et al. ${ }^{5)}$ reported healing of the menisci after repair appeared to be unrelated to the clinical improvement of symptoms. However, in the present study, meniscal healing seemed to be related to clinical symptomatic improvement: symptoms were improved in all patients in the complete healing group and good clinical outcomes were obtained in patients with incomplete meniscal healing (lax and scar healing). It is unclear why most patients demonstrated clinical improvement after root repair even though the structural integrity of the repaired meniscus was not observed to be restored.

The limitations of this study include the followings. First, because of its retrospective study design, anatomical data of the patients who did not have a second-look arthroscopy were not available; therefore, selection bias may have affected the results. Second, other factors that may influence the results were not evaluated, including postoperative compliance, patient's occupation and life style and time between root repair and second-look arthroscopy. Third, the cohort was small and most patients were female. Fourth, all the operations were performed using a single technique; therefore, the results may not be extrapolated to other techniques used for root repair. Fifth, the average follow-up pe$\operatorname{riod}(7$ months) was short. 


\section{Conclusions}

Arthroscopic pull-out suture repair is an effective method providing promising clinical results for patients with a symptomatic posterior root tear of the medial meniscus or even with a chronic degenerative root tear. However, it is unclear why clinical improvement occurred after root repair even in patients in whom structural integrity of the meniscus was not considered restored on second-look arthroscopy.

\section{Conflict of Interest}

No potential conflict of interest relevant to this article was reported.

\section{References}

1. Kenny C. Radial displacement of the medial meniscus and Fairbank's signs. Clin Orthop Relat Res. 1997;(339):163-73.

2. Lerer DB, Umans HR, Hu MX, Jones MH. The role of meniscal root pathology and radial meniscal tear in medial meniscal extrusion. Skeletal Radiol. 2004;33:569-74.

3. Bessette GC. The meniscus. Orthopedics. 1992;15:35-42.

4. Marzo JM, Gurske-DePerio J. Effects of medial meniscus posterior horn avulsion and repair on tibiofemoral contact area and peak contact pressure with clinical implications. Am J Sports Med. 2009;37:124-9.

5. Seo HS, Lee SC, Jung KA. Second-look arthroscopic findings after repairs of posterior root tears of the medial meniscus. Am J Sports Med. 2011;39:99-107.

6. Cho JH. Modified pull-out suture in posterior root tear of the medial meniscus: using a posteromedial portal. Knee Surg Relat Res. 2012;24:124-7.

7. Brittberg M, Winalski CS. Evaluation of cartilage injuries and repair. J Bone Joint Surg Am. 2003;85 Suppl 2:58-69.
8. Habata T, Uematsu K, Hattori K, Takakura Y, Fujisawa Y. Clinical features of the posterior horn tear in the medial meniscus. Arch Orthop Trauma Surg. 2004;124:642-5.

9. Ozkoc G, Circi E, Gonc U, Irgit K, Pourbagher A, Tandogan $\mathrm{RN}$. Radial tears in the root of the posterior horn of the medial meniscus. Knee Surg Sports Traumatol Arthrosc. 2008;16:849-54.

10. Bin SI, Kim JM, Shin SJ. Radial tears of the posterior horn of the medial meniscus. Arthroscopy. 2004;20:373-8.

11. Arnoczky SP, Warren RF. Microvasculature of the human meniscus. Am J Sports Med. 1982;10:90-5.

12. Choi NH, Son KM, Victoroff BN. Arthroscopic all-inside repair for a tear of posterior root of the medial meniscus: a technical note. Knee Surg Sports Traumatol Arthrosc. 2008;16:891-3.

13. Engelsohn E, Umans H, Difelice GS. Marginal fractures of the medial tibial plateau: possible association with medial meniscal root tear. Skeletal Radiol. 2007;36:73-6.

14. Kim YM, Rhee KJ, Lee JK, Hwang DS, Yang JY, Kim SJ. Arthroscopic pullout repair of a complete radial tear of the tibial attachment site of the medial meniscus posterior horn. Arthroscopy. 2006;22:795.

15. Griffith CJ, LaPrade RF, Fritts HM, Morgan PM. Posterior root avulsion fracture of the medial meniscus in an adolescent female patient with surgical reattachment. Am J Sports Med. 2008;36:789-92.

16. Ahn JH, Wang JH, Lim HC, Bae JH, Park JS, Yoo JC, Shyam AK. Double transosseous pull out suture technique for transection of posterior horn of medial meniscus. Arch Orthop Trauma Surg. 2009;129:387-92.

17. Lee JH, Lim YJ, Kim KB, Kim KH, Song JH. Arthroscopic pullout suture repair of posterior root tear of the medial meniscus: radiographic and clinical results with a 2 -year followup. Arthroscopy. 2009;25:951-8. 